\title{
Differential subordination for certain generalized operator
}

\author{
M. H. Al-Abbadi and M. Darus
}




\title{
DIFFERENTIAL SUBORDINATION FOR A CERTAIN GENERALIZED OPERATOR
}

\author{
M. H. AL-ABBADI AND M. DARUS
}

Received 16 February, 2011

\begin{abstract}
The authors have recently introduced a new generalized derivative operator $\mu_{\lambda_{1}, \lambda_{2}}^{n, m}$, that generalized many well-known operators. The trend of finding new differential or integral operators has attracted widespread interest. The aim of this paper is to use the relation

$$
(1+n) \mu_{\lambda_{1}, \lambda_{2}}^{n+1, m} f(z)=\left(\mu_{\lambda_{1}, \lambda_{2}}^{n, m} f(z)\right)^{\prime}+n\left(\mu_{\lambda_{1}, \lambda_{2}}^{n, m} f(z)\right)
$$

to discuss some interesting results by using the technique of differential subordination. The results include both subordination and inclusion. In the case of $n=0, \lambda_{2}=0$, we obtain the results of Oros [11].
\end{abstract}

2000 Mathematics Subject Classification: 30C45

Keywords: analytic function, Hadamard product (or convolution), univalent function; convex function, derivative operator, differential subordination, dominant, best dominant

\section{INTRODUCTION AND DEFINITIONS}

Let $\mathcal{A}$ denote the class of functions of the form

$$
f(z)=z+\sum_{k=2}^{\infty} a_{k} z^{k}
$$

which are analytic in the open unit disk $U=\{z \in \mathbb{C}:|z|<1\}$ on the complex plane $\mathbb{C}$. Let $S, S^{*}(\alpha), C(\alpha)(0 \leq \alpha<1)$ denote the subclasses of $\mathcal{A}$ consisting of functions that are univalent, starlike of order $\alpha$ and convex of order $\alpha$ in $U$, respectively. In particular, the classes $S^{*}(0)=S^{*}$ and $C(0)=C$ are the familiar classes of starlike and convex functions in $U$, respectively. And a function $f \in C(\alpha)$ if $\operatorname{Re}\left(1+\frac{z f^{\prime \prime}}{f^{\prime}}\right)>$ $\alpha$. Furthermore a function $f$ analytic in $U$ is said to be convex if it is univalent and $f(U)$ is convex.

Let $\mathscr{H}(U)$ be the class of holomorphic functions in unit disk $U=\{z \in \mathbb{C}:|z|<1\}$. Consider

$$
\mathcal{A}_{n}=\left\{f \in \mathscr{H}(U): f(z)=z+a_{n+1} z^{n+1}+\ldots ., \quad(z \in U)\right\}, \text { with } \mathcal{A}_{1}=\mathcal{A} .
$$


For $a \in \mathbb{C}$ and $n \in \mathbb{N}=\{1,2,3, \ldots$,$\} we let$

$$
\mathscr{H}[a, n]=\left\{f \in \mathcal{H}(U): f(z)=z+a_{n} z^{n}+a_{n+1} z^{n+1}+\ldots, \quad(z \in U)\right\} .
$$

Given two functions $f(z)=z+\sum_{k=2}^{\infty} a_{k} z^{k}$ and $g(z)=z+\sum_{k=2}^{\infty} b_{k} z^{k}$ analytic in the unit disk $U=\{z \in \mathbb{C}:|z|<1\}$, the Hadamard product (or convolution) $f * g$ is defined by

$$
f(z) * g(z)=(f * g)(z)=z+\sum_{k=2}^{\infty} a_{k} b_{k} z^{k} .
$$

Next, we state the basic ideas on subordination. If $f$ and $g$ are analytic in $U$, then the function $f$ is said to be subordinate to $g$, written as

$$
f \prec g \quad \text { or } \quad f(z) \prec g(z) \quad(z \in U),
$$

if and only if there exists the Schwarz function $w$, analytic in $U$, with $w(0)=0$ and $|w(z)|<1$ such that $f(z)=g(w(z)) \quad(z \in U)$.

Furthermore if $g$ is univalent in $U$, then $f \prec g$ if and only if $f(0)=g(0)$ and $f(U) \subset g(U)$. [see [14], p.36].

Let $\psi: \mathbb{C}^{3} \times U \rightarrow \mathbb{C}$ and let $h$ be univalent in $U$. If $p$ is analytic in $U$ and satisfies the (second-order) differential subordination

$$
\psi\left(p(z), z p^{\prime}(z), z^{2} p^{\prime \prime}(z) ; z\right) \prec h(z), \quad(z \in U),
$$

then $p$ is called a solution of the differential subordination.

The univalent function $q$ is called a dominant of the solutions of the differential subordination, or simply a dominant, if $p \prec q$ for all $p$ satisfying (1.2).

A dominant $\tilde{q}$ that satisfies $\tilde{q} \prec q$ for all dominants $q$ of (1.2) is said to be the best dominant of (1.2). (Note that the best dominant is unique up to a rotation of $U$ ).

Now, $(x)_{k}$ denotes the Pochhammer symbol (or the shifted factorial) defined by

$$
(x)_{k}= \begin{cases}1 & \text { for } k=0, x \in \mathbb{C} \backslash\{0\}, \\ x(x+1)(x+2) \ldots(x+k-1) \text { for } k \in \mathbb{N}=\{1,2,3, \ldots\} \text { and } x \in \mathbb{C} .\end{cases}
$$

In [1], the authors introduced and studied the generalized derivative operator $\mu_{\lambda_{1}, \lambda_{2}}^{n, m} f(z)$ given by the following definition.

Definition 1. For $f \in \mathcal{A}$ the generalized derivative operator $\mu_{\lambda_{1}, \lambda_{2}}^{n, m}$ is defined by $\mu_{\lambda_{1}, \lambda_{2}}^{n, m}: \mathcal{A} \rightarrow \mathcal{A}$,

$$
\mu_{\lambda_{1}, \lambda_{2}}^{n, m} f(z)=z+\sum_{k=2}^{\infty} \frac{\left(1+\lambda_{1}(k-1)\right)^{m}}{\left(1+\lambda_{2}(k-1)\right)^{m-1}} c(n, k) a_{k} z^{k}, \quad(z \in U),
$$

where $n, m \in \mathbb{N}_{0}=\{0,1,2 \ldots\}, \lambda_{2} \geq \lambda_{1} \geq 0$ and $c(n, k)=\left(\begin{array}{c}n+k-1 \\ n\end{array}\right)=\frac{(n+1)_{k-1}}{(1)_{k-1}}$. 
Special cases of this operator includes the Ruscheweyh derivative operator in two cases when $\mu_{0, \lambda_{2}}^{n, 1} \equiv R^{n}$ and $\mu_{\lambda_{1}, 0}^{n, 0} \equiv R^{n}$ [16], the Salagean derivative operator for $\mu_{1,0}^{0, m} \equiv S^{n}$ [17], the generalized Ruscheweyh derivative operator in the cases $\mu_{\lambda_{1}, \lambda_{2}}^{n, 1} \equiv R_{\lambda}^{n}$ and $\mu_{\lambda_{1}, \lambda_{2}}^{n, 0} \equiv R_{\lambda}^{n}$ [3], the generalized Salagean derivative operator introduced by Al-Oboudi $\mu_{\lambda_{1}, 0}^{0, m} \equiv S_{\beta}^{n}$ [2], and the generalized Al-Shaqsi and Darus derivative operator $\mu_{\lambda_{1}, 0}^{n, m} \equiv D_{\lambda, \beta}^{n}$ [5]. Now, let us recall the well known CarlsonShaffer operator $L(a, c)[4]$ associated to the incomplete beta function $\phi(a, c ; z)$, defined by

$$
\begin{aligned}
& L(a, c): \mathcal{A} \rightarrow \mathcal{A}, \\
& L(a, c) f(z):=\phi(a, c ; z) * f(z) \quad(z \in U), \\
& \text { where } \phi(a, c ; z)=z+\sum_{k=2}^{\infty} \frac{(a)_{k-1}}{(c)_{k-1}} z^{k} .
\end{aligned}
$$

It can be easily seen that

$$
\mu_{\lambda_{1}, 0}^{0,0} f(z)=\mu_{0, \lambda_{2}}^{1,0} f(z)=f(z)
$$

and

$$
\mu_{\lambda_{1}, 0}^{1,0} f(z)=\mu_{0, \lambda_{2}}^{1,1} f(z)=z f^{\prime}(z)
$$

Also $\mu_{\lambda_{1}, 0}^{a-1,0} f(z)=\mu_{0, \lambda_{2}}^{a-1,1} f(z)$ where $a=1,2,3, \ldots$.

To prove our results, we need the following equality:

$$
(1+n) \mu_{\lambda_{1}, \lambda_{2}}^{n+1, m} f(z)=z\left(\mu_{\lambda_{1}, \lambda_{2}}^{n, m} f(z)\right)^{\prime}+n\left(\mu_{\lambda_{1}, \lambda_{2}}^{n, m} f(z)\right), \quad(z \in U)
$$

where $n, m \in \mathbb{N}_{0}=\{0,1,2 \ldots\}$ and $\lambda_{2} \geq \lambda_{1} \geq 0$.

In addition, we need the following lemmas to prove our main results:

Lemma 1 ([9],p.71). Let $h$ be analytic, univalent, convex in $U$, with $h(0)=a$, $\gamma \neq 0$ and $\operatorname{Re} \gamma \geq 0$. If $p \in \mathscr{H}[a, n]$ and

$$
p(z)+\frac{z p^{\prime}(z)}{\gamma} \prec h(z), \quad(z \in U)
$$

then

$$
p(z) \prec q(z) \prec h(z), \quad(z \in U),
$$

where $q(z)=\frac{\gamma}{n z^{\frac{\nu}{n}}} \int_{0}^{z} h(t) t\left(\frac{\gamma}{n}\right)-1 d t, \quad(z \in U)$.

The function $q$ is convex and is the best $(a, n)$-dominant.

Lemma 2 ([8]). Let $g$ be a convex function in $U$ and let

$$
h(z)=g(z)+n \alpha z g^{\prime}(z)
$$

where $\alpha>0$ and $n$ is a positive integer.

If

$$
p(z)=g(0)+p_{n} z^{n}+p_{n+1} z^{n+1}+\ldots ., \quad(z \in U),
$$


is holomorphic in $U$ and

$$
p(z)+\alpha z p^{\prime}(z) \prec h(z), \quad(z \in U),
$$

then

$$
p(z) \prec g(z)
$$

and this result is sharp.

Lemma 3 ([10]). Let $f \in \mathcal{A}$, if

$$
\operatorname{Re}\left(1+\frac{z f^{\prime \prime}(z)}{f^{\prime}(z)}\right)>-\frac{1}{2}
$$

then

$$
\frac{2}{z} \int_{0}^{z} f(t) d t, \quad(z \in U \text { and } z \neq 0)
$$

is a convex function.

In the present paper, we shall use the method of differential subordination to derive certain properties of the generalized derivative operator $\mu_{\lambda_{1}, \lambda_{2}}^{n, m} f(z)$. Note that, differential subordination has been studied by various authors, and we follow the similar work of Oros [12] and Oros and Oros [13].

\section{MAIN RESULTS}

Before we state our first theorem, we give another definition.

Definition 2. For $n, m \in \mathbb{N}_{0}, \lambda_{2} \geq \lambda_{1} \geq 0$ and $0 \leq \alpha<1$, we let $R_{\lambda_{1}, \lambda_{2}}^{n, m}(\alpha)$ denote the class of functions $f \in \mathcal{A}$ which satisfy the condition

$$
\operatorname{Re}\left(\mu_{\lambda_{1}, \lambda_{2}}^{n, m} f(z)\right)^{\prime}>\alpha, \quad(z \in U) .
$$

It is clear that the class $R_{\lambda_{1}, 0}^{0,1}(\alpha) \equiv R\left(\lambda_{1}, \alpha\right)$ consists of functions $f \in \mathcal{A}$ satisfying

$$
\operatorname{Re}\left(\lambda_{1} z f^{\prime \prime}(z)+f^{\prime}(z)\right)>\alpha, \quad(z \in U),
$$

studied by Ponnusamy [15] and many others.

Now we begin with our first result.

Theorem 1. Let

$$
h(z)=\frac{1+(2 \alpha-1) z}{1+z}, \quad(z \in U),
$$

be convex in $U$, with $h(0)=1$ and $0 \leq \alpha<1$. If $n, m \in \mathbb{N}_{0}, \lambda_{2} \geq \lambda_{1} \geq 0$, and the differential subordination.

$$
\left(\mu_{\lambda_{1}, \lambda_{2}}^{n+1, m} f(z)\right)^{\prime} \prec h(z), \quad(z \in U),
$$


then

$$
\left(\mu_{\lambda_{1}, \lambda_{2}}^{n, m} f(z)\right)^{\prime} \prec q(z)=2 \alpha-1+\frac{2(n+1)(1-\alpha) \sigma(n)}{z^{n+1}},
$$

where $\sigma$ is given by

$$
\sigma(x)=\int_{0}^{z} \frac{t^{x}}{1+t} d t, \quad(z \in U)
$$

The function $q$ is convex and is the best dominant.

Proof. By differentiating (1.3), with respect to $z$, we obtain

$$
\left(\mu_{\lambda_{1}, \lambda_{2}}^{n+1, m} f(z)\right)^{\prime}=\frac{(1+n)\left(\mu_{\lambda_{1}, \lambda_{2}}^{n, m} f(z)\right)^{\prime}+z\left(\mu_{\lambda_{1}, \lambda_{2}}^{n, m} f(z)\right)^{\prime \prime}}{1+n}
$$

Using (2.4) in (2.2), differential subordination (2.2) becomes

$$
\begin{aligned}
\frac{(1+n)\left(\mu_{\lambda_{1}, \lambda_{2}}^{n, m} f(z)\right)^{\prime}+z\left(\mu_{\lambda_{1}, \lambda_{2}}^{n, m} f(z)\right)^{\prime \prime}}{1+n} & \prec h(z) \\
& =\frac{1+(2 \alpha-1) z}{1+z}
\end{aligned}
$$

Let

$$
\begin{aligned}
p(z)=\left[\mu_{\lambda_{1}, \lambda_{2}}^{n, m} f(z)\right]^{\prime} & =\left[z+\sum_{k=2}^{\infty} \frac{\left(1+\lambda_{1}(k-1)\right)^{m}}{\left(1+\lambda_{2}(k-1)\right)^{m-1}} c(n, k) a_{k} z^{k}\right]^{\prime} \\
& =1+p_{1} z+p_{2} z^{2}+\ldots . \quad(p \in \mathscr{H}[1,1], z \in U) .
\end{aligned}
$$

Using (2.6) in (2.5), the differential subordination becomes:

$$
p(z)+\frac{z p^{\prime}(z)}{1+n} \prec h(z)=\frac{1+(2 \alpha-1) z}{1+z} .
$$


By using Lemma 1, we have

$$
\begin{aligned}
p(z) \prec q(z) & =\frac{(n+1) \int_{0}^{z} h(t) t^{n} d t}{z^{n+1} z}, \\
& =\frac{(n+1) \int_{0}^{z}\left(\frac{1+(2 \alpha-1) t}{1+t}\right) t^{n} d t}{z^{n+1}}, \\
& =\frac{(n+1)\left[\sigma(n)+(2 \alpha-1) \int_{0}^{z} \frac{t^{n+1}}{1+t} d t\right],}{z^{n+1}[(n)}, \\
& =2 \alpha-1+\frac{2(n+1)(1-\alpha) \sigma(n)}{z^{n+1}},
\end{aligned}
$$

where $\sigma$ is given by (2.3), so we get

$$
\left[\mu_{\lambda_{1}, \lambda_{2}}^{n, m} f(z)\right]^{\prime} \prec q(z)=2 \alpha-1+\frac{2(n+1)(1-\alpha) \sigma(n)}{z^{n+1}} .
$$

The functions $q$ is convex and is the best dominant. The proof is complete.

Theorem 2. If $n, m \in \mathbb{N}_{0}, \lambda_{2} \geq \lambda_{1} \geq 0$ and $0 \leq \alpha<1$, then we have

$$
R_{\lambda_{1}, \lambda_{2}}^{n+1, m}(\alpha) \subset R_{\lambda_{1}, \lambda_{2}}^{n, m}(\delta)
$$

where

$$
\delta=2 \alpha-1+2(n+1)(1-\alpha) \sigma(n),
$$

where $\sigma$ is given by (2.3).

Proof. Let $f \in R_{\lambda_{1}, \lambda_{2}}^{n+1, m}(\alpha)$, then from (2.1) we have

$$
\operatorname{Re}\left(\mu_{\lambda_{1}, \lambda_{2}}^{n+1, m} f(z)\right)^{\prime}>\alpha, \quad(z \in U),
$$

which is equivalent to

$$
\left(\mu_{\lambda_{1}, \lambda_{2}}^{n+1, m} f(z)\right)^{\prime} \prec h(z)=\frac{1+(2 \alpha-1) z}{1+z} .
$$

Using Theorem 1, we have

$$
\left[\mu_{\lambda_{1}, \lambda_{2}}^{n, m} f(z)\right]^{\prime} \prec q(z)=2 \alpha-1+\frac{2(n+1)(1-\alpha) \sigma(n)}{z^{n+1}} .
$$

Since $q$ is convex and $q(U)$ is symmetric with respect to the real axis, we deduce

$$
\begin{aligned}
\operatorname{Re}\left[\mu_{\lambda_{1}, \lambda_{2}}^{n, m} f(z)\right]^{\prime}>\operatorname{Re} q(1) & =\delta=\delta\left(\alpha, \lambda_{1}\right) \\
& =2 \alpha-1+2(n+1)(1-\alpha) \sigma(n) .
\end{aligned}
$$


From that we deduce $R_{\lambda_{1}, \lambda_{2}}^{n+1, m}(\alpha) \subset R_{\lambda_{1}, \lambda_{2}}^{n, m}(\delta)$. This completes the proof of Theorem 2.

Theorem 3. Let $q$ be a convex function in $U$, with $q(0)=1$ and let

$$
h(z)=q(z)+\lambda_{1} z q^{\prime}(z), \quad(z \in U) .
$$

If $n, m \in \mathbb{N}_{0}, \lambda_{2} \geq \lambda_{1} \geq 0, f \in \mathcal{A}$ and it satisfies the differential subordination

$$
\left(\mu_{\lambda_{1}, \lambda_{2}}^{n+1, m} f(z)\right)^{\prime} \prec h(z), \quad(z \in U),
$$

then

$$
\left[\mu_{\lambda_{1}, \lambda_{2}}^{n, m} f(z)\right]^{\prime} \prec q(z), \quad(z \in U)
$$

and this result is sharp.

Proof. Let

$$
p(z)=\left(\mu_{\lambda_{1}, \lambda_{2}}^{n, m} f(z)\right)^{\prime} .
$$

Using (2.4), the differential subordination (2.7) becomes

$$
p(z)+\frac{z p^{\prime}(z)}{1+n} \prec h(z)=q(z)+\lambda_{1} z q^{\prime}(z), \quad(z \in U) .
$$

Using Lemma 2, we obtain

$$
p(z) \prec q(z), \quad(z \in U) .
$$

Hence

$$
\left[\mu_{\lambda_{1}, \lambda_{2}}^{n, m} f(z)\right]^{\prime} \prec q(z), \quad(z \in U) .
$$

The result is sharp. This completes the proof of the theorem.

We give a simple application for Theorem 3 .

Example 1. For $n=1, m=0, \lambda_{2} \geq \lambda_{1} \geq 0, q(z)=\frac{1+z}{1-z}, f \in \mathcal{A}$ and $z \in U$ and applying Theorem 3, we have

$$
h(z)=\frac{1+z}{1-z}+\lambda_{1} z\left(\frac{1+z}{1-z}\right)^{\prime}=\frac{1+2 \lambda_{1} z-z^{2}}{(1-z)^{2}} .
$$


By using (2.4) we find

$$
\begin{aligned}
\left(\mu_{\lambda_{1}, \lambda_{2}}^{1,0} f(z)\right)^{\prime}= & \left(\mu_{\lambda_{1}, \lambda_{2}}^{0,0} f(z)\right)^{\prime}+z\left(\mu_{\lambda_{1}, \lambda_{2}}^{0,0} f(z)\right)^{\prime \prime}, \\
= & 1+\sum_{k=2}^{\infty}\left(1+\lambda_{2}(k-1)\right) k a_{k} z^{k-1} \\
& +\sum_{k=2}^{\infty}\left(1+\lambda_{2}(k-1)\right) k(k-1) a_{k} z^{k-1}, \\
= & 1+\sum_{k=2}^{\infty}\left(1+\lambda_{2}(k-1)\right) k^{2} a_{k} z^{k-1}, \\
& f(z) *\left[z+\sum_{k=2}^{\infty}\left(1+\lambda_{2}(k-1)\right) k^{2} z^{k}\right] \\
z & \frac{f}{} .
\end{aligned}
$$

Similarly we compute $\left(\mu_{\lambda_{1}, \lambda_{2}}^{2,0} f(z)\right)^{\prime}$. By using (2.4), we find

$$
\left(\mu_{\lambda_{1}, \lambda_{2}}^{2,0} f(z)\right)^{\prime}=\left(\mu_{\lambda_{1}, \lambda_{2}}^{1,0} f(z)\right)^{\prime}+\frac{z}{2}\left(\mu_{\lambda_{1}, \lambda_{2}}^{1,0} f(z)\right)^{\prime \prime} .
$$

Then, by using (2.8) we have

$$
\left(\mu_{\lambda_{1}, \lambda_{2}}^{1,0} f(z)\right)^{\prime \prime}=\sum_{k=2}^{\infty}\left(1+\lambda_{2}(k-1)\right) k^{2}(k-1) a_{k} z^{k-2} .
$$

After that, by (2.8) and (2.10), (2.9) becomes

$$
\begin{aligned}
\left(\mu_{\lambda_{1}, \lambda_{2}}^{2,0} f(z)\right)^{\prime}= & 1+\sum_{k=2}^{\infty}\left(1+\lambda_{2}(k-1)\right) k^{2} a_{k} z^{k-1} \\
& +\frac{1}{2} \sum_{k=2}^{\infty}\left(1+\lambda_{2}(k-1)\right) k^{2}(k-1) a_{k} z^{k-1}, \\
= & 1+\sum_{k=2}^{\infty}\left(1+\lambda_{2}(k-1)\right) k^{2} \frac{(k+1)}{2} a_{k} z^{k-1}, \\
= & \frac{f(z) *\left[z+\sum_{k=2}^{\infty} \frac{1}{2}\left(1+\lambda_{2}(k-1)\right)(1+k) k^{2} z^{k}\right]}{z} .
\end{aligned}
$$


From Theorem 3 we deduce that

$$
\frac{f(z) *\left[z+\sum_{k=2}^{\infty} \frac{1}{2}\left(1+\lambda_{2}(k-1)\right)(1+k) k^{2} z^{k}\right]}{z} \prec \frac{1+2 \lambda_{1} z-z^{2}}{(1-z)^{2}}
$$

implies

$$
\frac{f(z) *\left[z+\sum_{k=2}^{\infty} k^{2}\left(1+\lambda_{2}(k-1)\right) z^{k}\right]}{z} \prec \frac{1+z}{1-z}, \quad,(z \in U) .
$$

Theorem 4. Let $q$ be a convex function in $U$, with $q(0)=1$ and let

$$
h(z)=q(z)+z q^{\prime}(z), \quad(z \in U) .
$$

If $n, m \in \mathbb{N}_{0}, \lambda_{2} \geq \lambda_{1} \geq 0, f \in \mathcal{A}$ and satisfies the differential subordination

$$
\left(\mu_{\lambda_{1}, \lambda_{2}}^{n, m} f(z)\right)^{\prime} \prec h(z),
$$

then

The result is sharp.

$$
\frac{\mu_{\lambda_{1}, \lambda_{2}}^{n, m} f(z)}{z} \prec q(z), \quad,(z \in U) .
$$

Proof.

$$
\begin{aligned}
p(z) & =\frac{\mu_{\lambda_{1}, \lambda_{2}}^{n, m} f(z)}{z}, \\
& =\frac{z+\sum_{k=2}^{\infty} \frac{\left(1+\lambda_{1}(k-1)\right)^{m}}{\left(1+\lambda_{2}(k-1)\right)^{m-1}} c(n, k) a_{k} z^{k}}{z}, \\
& =1+p_{1} z+p_{2} z^{2}+\ldots ., \quad(p \in \mathcal{H}[1,1], z \in U) .
\end{aligned}
$$

Differentiating (2.12), with respect to $z$, we obtain

$$
\left(\mu_{\lambda_{1}, \lambda_{2}}^{n, m} f(z)\right)^{\prime}=p(z)+z p^{\prime}(z), \quad(z \in U) .
$$

Using (2.13), the differential subordination (2.11) becomes

$$
p(z)+z p^{\prime}(z) \prec h(z)=q(z)+z q^{\prime}(z),
$$

and by using Lemma 2 , we deduce

$$
p(z) \prec q(z), \quad(z \in U) .
$$

Next using (2.12), we have

$$
\frac{\mu_{\lambda_{1}, \lambda_{2}}^{n, m} f(z)}{z} \prec q(z), \quad,(z \in U) .
$$


This proves Theorem 4 .

We give a simple application of Theorem 4.

Example 2. For $n=1, m=0, \lambda_{2} \geq \lambda_{1} \geq 0, q(z)=\frac{1}{1-z}, f \in \mathcal{A}$ and $z \in U$, by using Theorem 4, we obtain

$$
h(z)=\frac{1}{1-z}+z\left(\frac{1}{1-z}\right)^{\prime}=\frac{1}{(1-z)^{2}} .
$$

From (1.3), we have

$$
\begin{aligned}
\left(\mu_{\lambda_{1}, \lambda_{2}}^{1,0} f(z)\right) & =z\left(\mu_{\lambda_{1}, \lambda_{2}}^{0,0} f(z)\right)^{\prime} \\
& =z+\sum_{k=2}^{\infty}\left(1+\lambda_{2}(k-1)\right) k a_{k} z^{k} \\
& =f(z) *\left[z+\sum_{k=2}^{\infty}\left(1+\lambda_{2}(k-1)\right) k z^{k}\right] .
\end{aligned}
$$

From example 1, we have

$$
\left(\mu_{\lambda_{1}, \lambda_{2}}^{1,0} f(z)\right)^{\prime}=\frac{f(z) *\left[z+\sum_{k=2}^{\infty}\left(1+\lambda_{2}(k-1)\right) k^{2} z^{k}\right]}{z} .
$$

Now, applying Theorem 4, we deduce that

$$
\frac{f(z) *\left[z+\sum_{k=2}^{\infty}\left(1+\lambda_{2}(k-1)\right) k^{2} z^{k}\right]}{z} \prec \frac{1}{(1-z)^{2}}
$$

implies

$$
\frac{f(z) *\left[z+\sum_{k=2}^{\infty}\left(1+\lambda_{2}(k-1)\right) k z^{k}\right]}{z} \prec \frac{1}{1-z} .
$$

Theorem 5. Let

$$
h(z)=\frac{1+(2 \alpha-1) z}{1+z},(z \in U)
$$

be convex in $U$, with $h(0)=1$ and $0 \leq \alpha<1$. If $n, m \in \mathbb{N}_{0}, \lambda_{2} \geq \lambda_{1} \geq 0, f \in \mathcal{A}$ and the differential subordination

$$
\left(\mu_{\lambda_{1}, \lambda_{2}}^{n, m} f(z)\right)^{\prime} \prec h(z)
$$


is satisfied, then

$$
\frac{\mu_{\lambda_{1}, \lambda_{2}}^{n, m} f(z)}{z} \prec q(z)=2 \alpha-1+\frac{2(1-\alpha) \ln (1+z)}{z} .
$$

The function $q$ is convex and is the best dominant.

Proof. Let

$$
\begin{aligned}
p(z) & =\frac{\mu_{\lambda_{1}, \lambda_{2}}^{n, m} f(z)}{z}, \\
& =\frac{z+\sum_{k=2}^{\infty} \frac{\left(1+\lambda_{1}(k-1)\right)^{m}}{\left(1+\lambda_{2}(k-1)\right)^{m-1}} c(n, k) a_{k} z^{k}}{z}, \\
& =1+p_{1} z+p_{2} z^{2}+\ldots ., \quad(p \in \mathscr{H}[1,1], z \in U) .
\end{aligned}
$$

Differentiating (2.15), with respect to $z$, we obtain

$$
\left(\mu_{\lambda_{1}, \lambda_{2}}^{n, m} f(z)\right)^{\prime}=p(z)+z p^{\prime}(z), \quad(z \in U) .
$$

Using (2.16), the differential subordination (2.14) becomes

$$
p(z)+z p^{\prime}(z) \prec h(z)=\frac{1+(2 \alpha-1) z}{1+z}, \quad(z \in U) .
$$

From Lemma 1, we deduce

$$
\begin{aligned}
p(z) \prec q(z) & =\frac{1}{z} \int_{0}^{z} h(t) d t \\
& =\frac{1}{z} \int_{0}^{z}\left(\frac{1+(2 \alpha-1) t}{1+t}\right) d t \\
& =\frac{1}{z}\left[\int_{0}^{z} \frac{1}{1+t} d t+(2 \alpha-1) \int_{0}^{z} \frac{t}{1+t} d t\right], \\
& =2 \alpha-1+\frac{2(1-\alpha) \ln (1+z)}{z} .
\end{aligned}
$$

Using (2.15), we have

$$
\frac{\mu_{\lambda_{1}, \lambda_{2}}^{n, m} f(z)}{z} \prec q(z)=2 \alpha-1+\frac{2(1-\alpha) \ln (1+z)}{z} .
$$

The proof is complete.

From Theorem 5, we deduce the following Corollary: 
Corollary 1. If $f \in R_{\lambda_{1}, \lambda_{2}}^{n, m}(\alpha)$, then

$$
\operatorname{Re}\left(\frac{\mu_{\lambda_{1}, \lambda_{2}}^{n, m} f(z)}{z}\right)>(2 \alpha-1)+2(1-\alpha) \ln 2, \quad(z \in U) .
$$

Proof. Since $f \in R_{\lambda_{1}, \lambda_{2}}^{n, m}(\alpha)$, and from Definition 2 we have

$$
\operatorname{Re}\left(\mu_{\lambda_{1}, \lambda_{2}}^{n, m} f(z)\right)^{\prime}>\alpha, \quad(z \in U),
$$

which is equivalent to

$$
\left(\mu_{\lambda_{1}, \lambda_{2}}^{n, m} f(z)\right)^{\prime} \prec h(z)=\frac{1+(2 \alpha-1) z}{1+z} .
$$

Using Theorem 5, we have

$$
\frac{\mu_{\lambda_{1}, \lambda_{2}}^{n, m} f(z)}{z} \prec q(z)=(2 \alpha-1)+2(1-\alpha) \frac{\ln (1+z)}{z} .
$$

Since $q$ is convex and $q(U)$ is symmetric with respect to the real axis, we deduce

$$
\operatorname{Re}\left(\frac{\mu_{\lambda_{1}, \lambda_{2}}^{n, m} f(z)}{z}\right)>\operatorname{Re} q(1)=(2 \alpha-1)+2(1-\alpha) \ln 2, \quad(z \in U) .
$$

Theorem 6. Let $h \in \mathscr{H}(U)$, with $h(0)=1, h^{\prime}(0) \neq 0$ and assume that it satisfies the inequality

$$
\operatorname{Re}\left(1+\frac{z h^{\prime \prime}(z)}{h^{\prime}(z)}\right)>-\frac{1}{2}, \quad(z \in U) .
$$

If $n, m \in \mathbb{N}_{0}, \lambda_{2} \geq \lambda_{1} \geq 0, f \in \mathcal{A}$ and it satisfies the differential subordination

$$
\left(\mu_{\lambda_{1}, \lambda_{2}}^{n, m} f(z)\right)^{\prime} \prec h(z), \quad(z \in U),
$$

then

$$
\frac{\mu_{\lambda_{1}, \lambda_{2}}^{n, m} f(z)}{z} \prec q(z)=\frac{1}{z} \int_{0}^{z} h(t) d t
$$

Proof. Let

$$
\begin{aligned}
p(z) & =\frac{\mu_{\lambda_{1}, \lambda_{2}}^{n, m} f(z)}{z}, \\
& =\frac{z+\sum_{k=2}^{\infty} \frac{\left(1+\lambda_{1}(k-1)\right)^{m}}{\left(1+\lambda_{2}(k-1)\right)^{m-1}} c(n, k) a_{k} z^{k}}{z}, \\
& =1+p_{1} z+p_{2} z^{2}+\ldots ., \quad(p \in \mathscr{H}[1,1], z \in U) .
\end{aligned}
$$


Differentiating (2.18), with respect to $z$, we have

$$
\left(\mu_{\lambda_{1}, \lambda_{2}}^{n, m} f(z)\right)^{\prime}=p(z)+z p^{\prime}(z), \quad(z \in U) .
$$

Using (2.19), the differential subordination (2.17) becomes

$$
p(z)+z p^{\prime}(z) \prec h(z), \quad(z \in U) .
$$

From Lemma 1, we deduce

$$
p(z) \prec q(z)=\frac{1}{z} \int_{0}^{z} h(t) d t .
$$

With (2.18), we obtain

$$
\frac{\mu_{\lambda_{1}, \lambda_{2}}^{n, m} f(z)}{z} \prec q(z)=\frac{1}{z} \int_{0}^{z} h(t) d t .
$$

From Lemma 3, we obtain that the function $q$ is convex, and from Lemma 1, $q$ is the best dominant for the subordination (2.17). This completes the proof of Theorem 6.

\section{CONCLUSION}

We remark that several subclasses of analytic univalent functions can be derived using the operator $\mu_{\lambda_{1}, \lambda_{2}}^{n, m}$. Several of their properties can be studied with this method, for example properties related to the ones that were studied in [7] and [6].

\section{ACKNOWLEDGEMENT}

This work was supported by UKM-ST-06-FRGS0244-2010, Malaysia. The authors would like to thank the referee for giving some suggestions to improve the content of the article.

\section{REFERENCES}

[1] M. H. Al-Abbadi and M. Darus, "Differential subordination defined by new generalised derivative operator for analytic functions," Int. J. Math. Math. Sci., vol. 2010, p. 15, 2010.

[2] F. M. Al-Oboudi, "On univalent functions defined by a generalized Sălăgean operator," Int. J. Math. Math. Sci., vol. 2004, no. 25-28, pp. 1429-1436, 2004.

[3] K. Al-Shaqsi and M. Darus, "On univalent functions with respect to $k$-symmetric points defined by a generalized Ruscheweyh derivatives operator," J. Anal. Appl., vol. 7, no. 1, pp. 53-61, 2009.

[4] B. Carlson and D. B. Shaffer, "Starlike and prestarlike hypergeometric functions," SIAM J. Math. Anal., vol. 15, pp. 737-745, 1984.

[5] M. Darus and K. Al-Shaqsi, "Differential sandwich theorems with generalised derivative operator," Int. J. Comput. Math. Sci., vol. 2, no. 2, pp. 75-78, 2008.

[6] M. Darus and I. Faisal, "A study of Pescar's univalence criteria for space of analytic functions," Journal of Inequalities and Applications, vol. 2011, no. 109, p. 7, 2011. 
[7] M. Darus and I. Faisal, "A study on Becker's univalence criteria," Abstr. Appl. Anal., vol. 2011, p. 13, 2011.

[8] S. S. Miller and P. T. Mocanu, "On some classes of first-order differential subordinations," Mich. Math. J., vol. 32, pp. 185-195, 1985.

[9] S. S. Miller and P. T. Mocanu, Differential subordinations: theory and applications, ser. Series on monographs and textbooks in pure and applied mathematics. New York: Marcel Dekker, 2000, vol. 225.

[10] P. T. Mocanu, T. Bulboaca, and G. S. Salagean, Teoria geometrica a functiilor univalente. ClujNapoca: Casa Cartii de Stiinta, 1999.

[11] G. I. Oros, "On a class of holomorphic functions defined by the Ruscheweyh derivative," Int. J. Math. Math. Sci., vol. 2003, no. 65, pp. 4139-4144, 2003.

[12] G. I. Oros, "A class of holomorphic functions defined using a differential operator," Gen. Math., vol. 13, no. 4, pp. 13-18, 2005.

[13] G. Oros and O. G. Irina, "Differential superordination defined by Sălăgean operator," Gen. Math., vol. 12, no. 4, pp. 3-10, 2004.

[14] K. S. Padmanabhan and R. Manjini, "Certain applications of differential subordination," Publ. Inst. Math., Nouv. Sér., vol. 39, no. 53, pp. 107-118, 1986.

[15] S. Ponnusamy, "Differential subordination and starlike functions," Complex Variables, Theory Appl., vol. 19, no. 3, pp. 185-194, 1992.

[16] S. Ruscheweyh, "New criteria for univalent functions," Proc. Am. Math. Soc., vol. 49, pp. 109$115,1975$.

[17] G. S. Sălăgean, "Subclasses of univalent functions," in Complex analysis - Proc. 5th Rom.-Finn. Semin., Bucharest 1981, ser. Lect. Notes Math., vol. 1013. Springer-Verlag, 1983, pp. 362-372.

\section{Authors' addresses}

\section{H. Al-Abbadi}

Universiti Kebangsaan Malaysia, School of Mathematical Sciences, Faculty of Science and Technology, Bangi 43600, Selangor, Malaysia

E-mail address: mamoun_nn@yahoo.com

\section{Darus}

Universiti Kebangsaan Malaysia, School of Mathematical Sciences, Faculty of Science and Technology, Bangi 43600, Selangor, Malaysia

E-mail address: maslina@ukm.my 